\title{
Youth-adult partnership: exploring contributions to empowerment, agency and community connections in Malaysian youth programs
}

\begin{abstract}
Youth-adult partnership (Y-AP) has emerged as a key practice for enacting two features of effective developmental settings: supportive adult relationships and support for efficacy and mattering. Previous studies have shown that when youth, supported by adults, actively participate in organizational and community decision-making they are likely to show greater confidence and agency, empowerment and critical consciousness, and community connections. Most of the extant research on Y-AP is limited to qualitative studies and the identification of organizational best practices. Almost all research focuses on Western sociocultural settings. To address these gaps, 299 youth, age 15 to 24, were sampled from established afterschool and community programs in Malaysia to explore the contribution of Y-AP (operationalized as having two components: youth voice in decision-making and supportive adult relationships) to empowerment, agency and community connections. As hypothesized, hierarchical regressions indicated that program quality (Y-AP, safe environment and program engagement) contributed to agency, empowerment and community connections beyond the contribution of family, school and religion. Additionally, the Y-AP measures contributed substantially more variance than the other measures of program quality on each outcome. Interaction effects indicated differences by age for empowerment and agency but not for community connections. The primary findings in this inquiry replicate those found in previous interview and observational-oriented studies. The data suggests fertile ground for future research while demonstrating that Y-AP may be an effective practice for positive youth development outside of Western settings.
\end{abstract}

Keyword: Adolescents; Youth-adult partnership; Positive youth development; Community connections; Personal agency; Psychological empowerment 